5 heures. Avec un peu d'habitude, on arrive à siphonner la presque totalité de la couche éthérée, ce qui dispense de l'emploi de tubes gradués.

Dosage des cendres. - Lincinération se fait sur 3 grammes de caséine dans une eapsule de platine tarée et recouverte au début pour éviter les pertes dues aux projections ou au boursouflement. On ne dépasse pas le rouge sombre et on arrête l'opération quand les cendres sont blanches. L'opération est relativement rapide avec les caséines à la présure; elle est beaucoup plus lente avec les easéines lactiques.

\title{
NOTE SUR LE DOSAGE DE LA MATIËRE GRASSE DANS LA CASÉINE
}

\section{par Mare FOUASSIER,}

En ce qui concerne le taux de matière grasse dans les caséines, il est indispensable qu'une entente internationale intervienne. Beaucoup de marchés pour l'exportation reposent sur ce taux, et les méthodes utilisées pour le déterminer ne donnent pas des résultats concordants.

En France, on semble s'en tenir à la méthode par épuisement avec des solvants appropriés, en utilisant la Soxhlet. Cette méthode est longue et son résultat toujours inférieur à celui que l'on devrait obtenir réellement. La grosseur du grain, la pénétration aux solvants en font une cause d'insuccès.

Je signale pour mémoire le broyage au sable de la caséine préalablement humidifiée ; là encore, l'extraction est incomplète.

Seules, les méthodes basées sur la désagrégation préalable ét totale du grain de caséine, dans un milieu acide ou alcalin, suivie de l'extrac. tion de la matière grasse de ce milieu, par les solvants appropriés, permettent le dosage certain et régulier de la totalité de la matière grasse d'une caséine.

En Allemagne, on utilise la méthode Gottlieb-Ratzlaff, ou la méthode Schmid-Bondzynski-Ratzlaff, l'une et l'autre utilisant la désagrégation en milieu acide. La dernière de ces méthodes est décrite dans les Annales des Falsifications et des Fraudes, no 127 , page 41 , où elle est appliquée aux fromages.

Appelé à faire de nombreuses analyses de caséines destinées à l'exportation, j'utilisais pour le dosage de la matière grasse, la méthode par extraction au Soxhlet, mes résultats étaient toujours très sensiblement différents de ceux obtenus en Allemagne, par exemple, lors de la contreanalyse, ce qui a donné lieu à de multiples contestations. Il m'est apparu nécessaire de demander communication de la méthode utilisée, et par suite, de l'employer moi-même, il en est résulté une complète concordancenentre les résultats obtenus de part et d'autre et, par conséquent, la disparition de toute discussion. La méthode indiquée est celle de Gottlieb-Ratzlaff. 
Je crois intéressant de communiquer au Congrès pour l'Avancement des Sciences les modifications d'ordre opératoire apportées par moi à la méthode Gottlieb-Ratzlaff; ; elles permettent d'obtenir de cette méthode plus de rapidité et de précision.

Technique. - Voici la technique de la méthode Gottlieb. Ratzlaff : Dans un verre de Jena, on pèse 5 gr. de caséine, que l'on dissout totalement et à chaud dans 15 cent. cube d'acide chlorydrique de Dté. 1,125. On transvase ensuite dans un tube Gottlieb-Roese, ou une éprouvette graduée de $100 \mathrm{cc}$. On rince le verre de Jena avec $100 \mathrm{cc}$. d'alcool, puis $25 \mathrm{cc}$. d'éther et enfin $25 \mathrm{cc}$. d'éther de pétrole.

Chaque liquide de lavage est versé dans l'éprouvette qui est ensuite couchée, puis agitée. On laisse alors reposer pendant deux heures. On prélève $10 \mathrm{cc}$. de la partie éthérée surnageante sur laquelle on effectue par évaporation et pesée le dosage de la matière grasse, après avoir naturellement au préalable, mesuré le volume total.

Critique. - L'attaque en vase ouvert est une cause d'émission de vapeurs acides intenses dans le laboratoire. Le transvasement et le rinçage sont une perte de temps et une cause d'erreur. Le repos de deux heures nécessité pour la séparation complète des couches, prolonge de beaucoup l'analyse.

Technique modifiée et proposée par M. Fouassier. - J'ai fait établir en verre Pyrex, des tubes gradués en demi co. dans lesquels on introduit 2 gr. 5 de caséine exactement pesés. On ajoute dans le tube $5 \mathrm{cc}$. d'acide chlorhydrique de Dté 1,125 , on adapte un bouchon muni d'un tube à dégagement avec boule et pointe effilée, dont l'extrémité plonge dans une solution alcaline, le tout est porté au B. M. bouillant. Au bout de 20 minutes environ, la dissolution est complète, sans émission extérieure de vapeurs acides. On refroidit le tube, puis on ajoute $5 \mathrm{cc}$. d'alcool, $10 \mathrm{ce}$. d'éther et $10 \mathrm{cc}$. d'éther de pétrole. On agite le tube bouché, puis on le porte dans la centrifugeuse Gerber (sa construction ayant été conçue pour qu'il s'y adapte). Après une courte centrifugation, la séparation des couches est totale et complète, la graduation du tube permet de lire immédiatement la hauteur de la couche éthérée que l'on note, et dont on prélève ensuite $10 \mathrm{cc}$. que l'on évapore sèche et pèse. On rapporte le poids trouvé au volume total de solution éthérée pour $2 \mathrm{gr}$. 5 de caséine, le résultat est donné pour cent.

Dans la méthode que je préconise, et que j'utilise avec toute satisfaction, grâce aux tubes que j'ai fait construire spécialement, on remar: quera une simplification dans les manipulations, une économie de temps et de solvants employés. L'absorption des vapeurs acides n'est également pas négligeable.

A titre comparatif, j'ai essayé la méthode de MM. Tapernoux, Desrante et Binead, mentionnée dans la Revue Le Lait $\mathrm{n}^{\circ} 77$, cette 
méthode m'a semblé moins rapide, moins précise à cause des transvasements et de la petite quantité de caséine mise en œuvre ; en outre, on risque d'entraîner beaucoup plus aisément du non-beurre dans les décantations, que lorsqu'il s'agit de pipeter un liquide éthéré limpide.

\section{BIBLIOGRAPHIE ANALYTIQUE}

\section{LES LIVRES}

\section{Congrès International de Protection de l'Enfance. - Paris, juillet 1928. 2 volumes.}

Ce travail important, deux volumes dont le total fait 782 pages, contient les rapports qui ont été présentés à ce Congrès, lequel comprenait diverses sections : Maisons maternelles, Etude comparée des moyens les meilleurs pour développer l'allaitement maternel, les Etablissements de plein air pour les enfants, Le Service social dans la protection de l'Enfance.

Tous ces rapports sont extrêmement intéressants et méritent d'être lus attentivement.

Ce Congrès n'a pas voulu aborder la question de l'alimentation artificielle des enfants estimant qu'il importait avant tout de faire porter son effort dans la section correspondante : sur les moyens qu'il fallait mettre en ceuvre pour développer l'allaitement maternel.

On ne saurait, évidemment, trop féliciter ceux qui sont à la tête d'un pareil mouvement, mais on doit se demander parfois si leurs efforts et leurs désirs reçoivent leur récompense, comme ils le mériteraient.

Les conséquences funestes de la guerre qui ont conduit la femme plus que jamais, malheureusement, à abandonner son foyer, ont retenti fâcheusement sur l'allaitement au sein.

C'est ainsi qu'un délégué polonais, dans son rapport, nous eitait des chiffres déconcertants : alors qu'avant la guerre l'allaitement au sein répondait à $95 \%$ des enfants, depuis la guerre cette proportion s'est abaissée à $55 \%$.

II est done bien de faire tout ce qui peut être fait pour que l'enfant reçoive le sein maternel. Peut-être n'est-il pas moins bien, devant les problèmes sociaux qui se posent, de chercher à fournir à l'enfant, qui ne reçoit pas le sein, une meilleure alimentation.

Ch. PORCHER.

\section{Bibliography of Scientific Periodical Literature. - Section: Milk and Dairy Science.}

Cataloguer des travaux scientifiques est certainement un des devoirs du bibliothécaire, mais c'èst un devoir des plus coûteux et des plus difficiles à remplir 